\title{
Meningoencephelocele through the lateral craniopharyngeal canal
}

\begin{abstract}
Meningoencephelocele is a neural tube defect which manifests in early infancy and characterized by sac like protrusions of the brain parenchyma and it's covering membranes which prolapsed through the skull base defect. Here we report a rare case of meningoencephelocele via the Sternberg's canal and entering the sphenoid sinus through the onodi cell. A 53year old presented to our outpatient department with complaints of clear watery discharge from the left nasal cavity for the past one and a half months. DNE showed an active CSF leak from the sphenoid recess. MR Cisternography confirmed the diagnosis of meningoencepholecele. Patient was managed with endoscopic excision of meningoencephelocele and closure of skull base defect. Patient is disease free on follow up.
\end{abstract}

Keywords: meningoencephelocele, sternberg's canal, onodi cell
Volume 3 Issue 3 - 2015

Somu L, Prasanna Kumar, Prem Nivas R

Department of ENT, Head and neck surgery, Sri Ramachandra University, India

Correspondence: Somu L, Dept of Ent, Head and neck surgery, Sri Ramachandra University, India, Tel +9l 984004I597, Email premnivasar@ymail.com

Received: January 15, 2015 | Published: September 24, 2015

\section{Introduction}

Meningoencephelocele is a neural tube defect which manifests in early infancy and characterized by sac like protrusions of the brain parenchyma and it's covering membranes which prolapsed through the skull base defect. Sphenoid sinus CSF leaks, especially when associated with a concomitant meningoencepholecele, are thought to usually involve Sternberg's canal which is a persistent lateral craniopharyngeal canal. ${ }^{1}$ Here, we present a case of a meningoencephalocele which was seen to be involving both the sternberg's canal and the Onodi cell, which has an incidence of $8-14 \%$ of all sphenoid sinus leaks. ${ }^{2,3}$ According to the Anatomic Terminology Group, Onodi cells can be identified when posterior ethmoid cells become pneumatised laterally and to some extent superior to the sphenoid sinus and are usually close to the optic nerve. ${ }^{4}$ Embryologically, they are derived from ethmoid cells that have undergone dedifferentiation. Due to its relationship with the optic nerve, the internal carotid artery and cellar floor, the Onodi cells make for an important anatomical variant during sphenoidal sinus surgery. ${ }^{5}$ In 1888 Maxmillan Sternberg identified a defect in the lateral wall of the sphenoid sinus, a bony-cartilaginous one and described it as 'lateral cricopharyngeal canal'. Since then many variations of the lateral craniopharyngeal canal have been documented in literature. ${ }^{6}$

\section{Case report}

A 53year old gentleman came to the OPD with chief complaints of clear watery discharge from the left nasal cavity for the past one and a half months. Examination showed clear watery discharge when the patient bent forward indicative of cerebrospinal fluid. Diagnostic nasal endoscopy showed an active CSF leak from the sphenoid recess. CT of paranasal sinuses showed mucosal thickening in the left side of the sphenoid sinus and left posterior ethmoidal sinus with obstruction of sphenoethmoidal recess (Figure 1). MRI Cisternography showed evidence of bony defect in the left lateral wall of the sphenoid sinus measuring $9 \times 10 \mathrm{~mm}(\mathrm{CC} \times \mathrm{AP})$ with hernia ion of the small portion of the medial left temporal lobe with dura \& CSF- suggestive of left medial temporal lobe encephalocele (Figure $2 \& 3$ ).

Based on the radiological findings, surgery with the aid of a nasal endoscope was done under general anesthesia. The sphenoid ostium was identified, and meningoencephalocele was seen in the sphenoid sinus. Stripping off the mucosa lining the sphenoidal sinus was done in order to prevent the formation of mucocele later, and the menigoencephalocele was cauterized at the pedicle and removed. The mass was seen to be involving the sphenoid sinus through the Sternberg's canal and involving the Onodi cell. The defect was plugged with fascia lata and fat along with tissue glue. Whole of sphenoid sinus was obliterated with fat, fascia, surgical gel and tissue gel, and was reinforced with had ad flap.post operatively antibiotic coverage was given. After two days Nasal pack was removed and patient was discharged. One week later patient was reviewed with endoscopy which showed no signs of CSF leak. The patient shows no signs of recurrence, lyear after surgery. The patient is still on regular follow up.

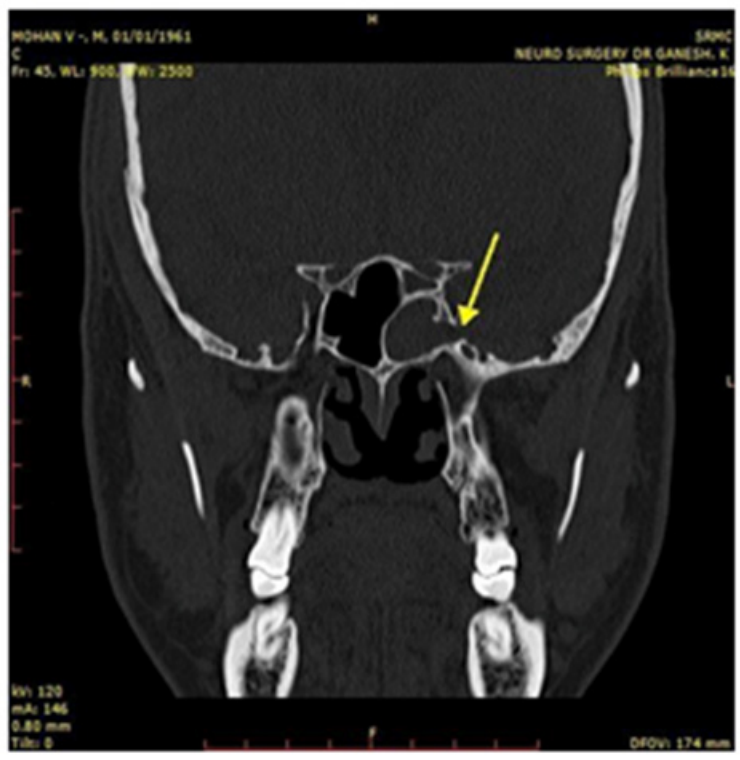

Figure I CT PNS showing homogenous soft tissue shadow in the left sphenoid sinus. The arrow showing the area of the Sternberg's canal.

\section{Discussion}

Meningoencephelocele is a neural tube defect which manifests in early infancy and characterized by sac like protrusions of the brain 
parenchyma and it's covering membranes which prolapsed through the skull base defect. When the herniated sac is composed of only the meanings and cerebrospinal fluid it is referred to as meningocele. If the herniated sac also includes the brain parenchyma it is termed as meningoencephalocele. Meningoencepholecele has specific characteristic findings like pulsation, Furstenberg sign (distend with crying or compression over the jugular vein) and a defect in the skull base. In certain cases where the mass is fibrous, the above said findings are absent. It can be misdiagnosed to be a nasal polyp; polyps are usually non-pulsatile, pinkish masses and are located lateral to the middle turbinate. In the contrary, meningoencephelocele will be shiny and turbid and located between the middle turbinate and septum. The significance of the posterior ethmoid cells was first described by Adolf Onodi et al., ${ }^{7}$ Whereas literature showed an incidence of the Onodi cell of 3.4-51\%, ${ }^{8}$ more recent studies show it to be $8-14 \%{ }^{2,3}$ Its location is mainly superolateral to the sphenoid sinus, and because of its close relation to the optic nerve, there is increased risk of nerve injury when any sort of pathology is associated with this space. Optic neuropathy is a dreaded complication associated with mucocele, due to compression of the optic nerve since it passes within the Onodi cell and it has been described in the literature. ${ }^{9,10}$ It can be inferred from this that the meningoencephalocele in our patient may have had the potential of causing an optic neuropathy based on the anotomical location of the involvement. Sphenoid sinus cerebrospinal fluid leaks are less common than leaks from the ethmoid or cribriform plate region ${ }^{11}$ Moreover; they pose a challenge in terms of visualization and access. However, minimally invasive endoscopic repair may be accomplished with an acceptable rate of morbidity and excellent outcomes. When combining series of CSF leak repair in literature, there is a successful+ initial repair in 55 of the 58 cases (95\%). Various surgical techniques have been introduced; however the main thing is to prevent complication and recurrence while removing the mass. Endoscopic removal of meningoencephalocele and reconstruction of the defect is done since endoscope provides an expanded and clear image, therefore the surgical visual field is very accurate and the defect in the skull base can be precisely located, so that the graft can be accurately placed into the defect.
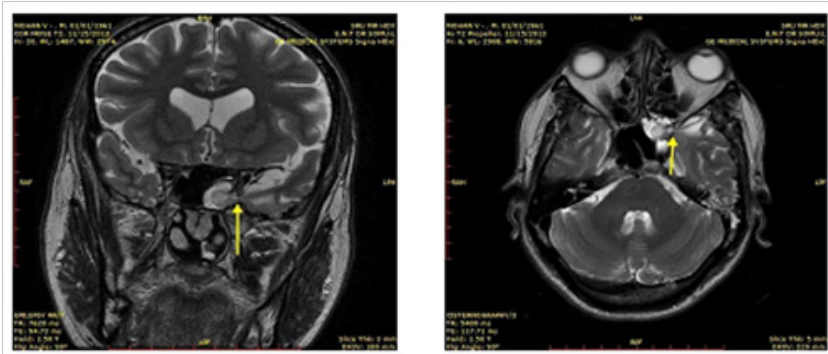

Figure 2\&3 MRI Cisternography pictures showing defect in the left side of the sphenoid sinus with hernia ion of small portion of the left temporal lobe with dura \& CSF.

In our patient, watery nasal discharge from the nose prompted us to do diagnostic nasal endoscopy and MRI cisternography which confirmed the diagnosis of meningoencephelocele via the Sternberg's canal and entering the sphenoid sinus through the Onodi cell. Endoscopic surgical repair of the leak resulted in successful treatment. The repair not only allowed us to arrest the CSF leak and prevent sequelae of meningitis, intracranial abscess, and pneumocephalus, ${ }^{11}$ but also visualize the extent of involvement, identify an important anatomical variant (Onodi cell), and prevent any risk of optic neuropathy.

\section{Acknowledgments}

None.

\section{Conflicts of interest}

Author declares there are no conflicts of interest.

\section{Funding}

None.

\section{References}

1. Schick, Bernhard, Dominik Brors, et al. Sternberg's canal-cause of congenital sphenoidal meningocele. Eur arc otorhinolaryngol. 2000;257(8):430-432.

2. Weinberger, Debra G, Anand, et al. Surgical anatomy and variations of the Onodi cell. American Journal of Rhinology. 1996;10(6):365-370.

3. Jones, NS, Strobl A, Holland I. A study of the CT findings in 100 patients with rhinosinusitis and 100 controls. Clin Otolaryngol Allied Sci. 1997;22(1):47-51

4. Stammberger H, David WK, Bolger W. Paranasal sinuses: anatomic terminology and nomenclature. Ann Otol Rhinol Laryngol. $1995 ; 167: 17-21$.

5. Shin, Hyeon ji, Hong YK, et al. The Onodi Cell an Obstacle to Sellar Lesions with a Transsphenoidal Approach. Otolaryngol Head Neck Surg. 2011;145(6):1040-1042.

6. Sterneberg M. Ein bisher noch nicht beschriebener Kanal im Keilben des Menschen. Anat Anz. 1888;3:225-253.

7. Onodi A. Die Sehstörungen und Erblindung nasalen Ursprunges, bedingt durch Erkrank-ungen der hinteren Nebenhöhlen. Z Augenheilkd. 1904;12(1):23-46.

8. Dixon, FW. A comparative study of the sphenoid sinus (a study of 1600 skulls). Ann Otol Rhinol Laryngol. 1937;46:687-698.

9. T Klink, J Pahnke, F Hoppe, et al. Acute visual loss by an Onodi cell. The Br ophthalmol. 2000;84(7):799.

10. Ogata, Yoichi, Yoshihiko Okinaka, et al. Optic neuropathy caused by an isolated mucocele in an Onodi cell. ORL. 1998;60(6):349-352.

11. Sautter, NB, Pete Batra S, MJ, Citardi . Endoscopic management of sphenoid sinus cerebrospinal fluid leaks. Anna otol rhinol Laryngol. 2008;117(1):32-39. 